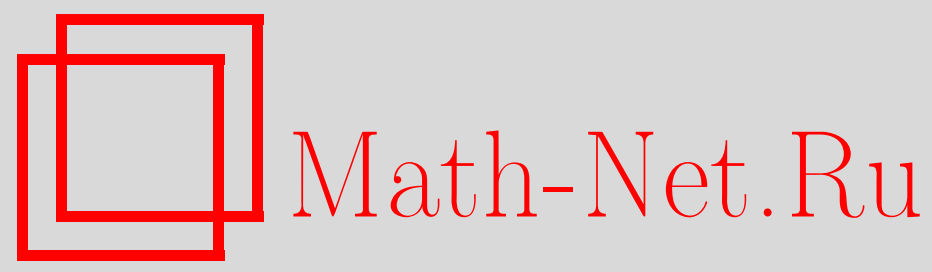

А. И. Перов, Теорема Йорка и неравенство Виртингера, $M a$ тем. заметки, 2001, том 70, выпуск 2, 237-245

DOI: https://doi.org/10.4213/mzm737

Использование Общероссийского математического портала Math-Net.Ru подразумевает, что вы прочитали и согласны с пользовательским соглашением http://www . mathnet.ru/rus/agreement

Параметры загрузки:

IP: 35.173 .137 .237

26 апреля 2023 г., 18:05:43






\section{ТЕОРЕМА ЙОРКА И НЕРАВЕНСТВО ВИРТИНГЕРА}

\section{А. И. Перов}

Рассматриваются автономные системы обыкновенных дифференциальных уравнений (первого или болеевысокого порядка), правые части которых удовлетворяют условию Липшица, формулируемому с помошью евклидовой метрики и неотрицательных матриц. На основе неравенства Виртингера доказаны теоремы об оценке снизу периода периодических нестационарных решений автономных систем, обобщающие теорему Йорка. В случае неотрицательных неразложимых матриц обсуждается вопрос о точности полученньх оценок.

Библиограбфия: 5 названий.

Статья посвящена получению оценок снизу для не сводящихся к постоянным периодов периодических решений автономных систем дифференциальных уравнений, правые части которых удовлетворяют условию Липшица, сформулированному с помощњю евклидовой нормы и неотрицательных матриц.

Для $n$-мерных векторов $\boldsymbol{x}$ и $\boldsymbol{y}$ с числовыми компонентами $x_{1}, \ldots, x_{n}$ и $y_{1}, \ldots, y_{n}$ соответственно скалярное произведение и евклидову норму определяем обычным образом:

$$
(\boldsymbol{x}, \boldsymbol{y})=\sum_{j=1}^{n} \bar{x}_{j} y_{j}, \quad\|\boldsymbol{x}\|=\left(\sum_{j=1}^{n}\left|x_{j}\right|^{2}\right)^{1 / 2}
$$

Рассмотрим записанную в векторной форме автономную систему нелинейных дифференциальных уравнений

$$
\dot{\boldsymbol{x}}_{j}=\boldsymbol{g}_{j}\left(\boldsymbol{x}_{1}, \ldots, \boldsymbol{x}_{m}\right), \quad j=1, \ldots, m,
$$

где точка означает дифференцирование по времени $t, \boldsymbol{x}_{j}$ - вектор размерности $d_{j}$, причем $d_{1}+\cdots+d_{m}=n$. В вещественном случае $\boldsymbol{g}_{j}\left(\boldsymbol{x}_{1}, \ldots, \boldsymbol{x}_{m}\right)$ определены для $\boldsymbol{x}_{1} \in \mathbb{R}^{d_{1}}, \ldots, \boldsymbol{x}_{m} \in \mathbb{R}^{d_{m}}$ и принимают значения в $\mathbb{R}^{d_{j}}$, в комплексном случае $\boldsymbol{g}_{j}\left(\boldsymbol{x}_{1}\right.$, $\left.\ldots, \boldsymbol{x}_{m}\right)$ определены для $\boldsymbol{x}_{1} \in \mathbb{C}^{d_{1}}, \ldots, \boldsymbol{x}_{m} \in \mathbb{C}^{d_{m}}$ и принимают значения в $\mathbb{C}^{d_{j}}(j=1$, $\ldots, m)$. Решение $\boldsymbol{x}(t)$ системы $(1)$ с векторньми компонентами $\boldsymbol{x}_{1}(t), \ldots, \boldsymbol{x}_{m}(t)$ назовем стационарным, если все его компоненты являются постоянньпи, и нестационарнымв противном случае. Нас будут интересовать периодические нестационарные решения, т.е. решения, для которых

$$
\boldsymbol{x}_{j}(t+\omega)=\boldsymbol{x}_{j}(t), \quad t \in \mathbb{R}, \quad j=1, \ldots, m,
$$


где период $\omega$ - некоторое положительное число.

В статье важную роль играют неотрицательные векторы и матрищы [1]. Спектральны й радиус квадратной матрицы $A$ обозначается spr $A$. Предполагается, что выполнено условие Липшица в следуюшей форме:

$$
\left\|\boldsymbol{g}_{j}\left(\boldsymbol{x}_{1}, \ldots, \boldsymbol{x}_{m}\right)-\boldsymbol{g}_{j}\left(\boldsymbol{y}_{1}, \ldots, \boldsymbol{y}_{m}\right)\right\| \leqslant \sum_{k=1}^{m} l_{j k}\left\|\boldsymbol{x}_{k}-\boldsymbol{y}_{k}\right\|, \quad j=1, \ldots, m,
$$

где $\boldsymbol{L}=\left(l_{j k}\right)$ - неотрицательная $(m \times m)$-матрица, спектральньй радиус которой положителен: spr $\boldsymbol{L}>0$. Ясно, что в силу условия (3) фазовая траектория $\boldsymbol{x}(t)$ в $n$-мерном фазовом пространстве не может слишком быстро вернуться назад на прежнее место; иными словами, период $\omega$ в (2) не может быть слишком малым (если только решение не сводится к стационарному). Количественное подтверждение отмеченного явления содержится в приводимой ниже теореме 1 , которая в вещественном случае и при дополнительном предположении $m=1$ впервые была установлена Йорком [2].

В теореме Йорка система (1) в $\mathbb{R}^{n}$ имеет вид $\dot{\boldsymbol{x}}=\boldsymbol{g}(\boldsymbol{x})$, где выполнено условие Липшица $\|\boldsymbol{g}(\boldsymbol{x})-\boldsymbol{g}(\boldsymbol{y})\| \leqslant l\|\boldsymbol{x}-\boldsymbol{y}\|$ с положительной постоянной $l$, а оценка периода состоит в том, что $\omega \geqslant 2 \pi / l$.

Для вещественных векторов $\boldsymbol{x}$ и $\boldsymbol{y}$ мы пишем $\boldsymbol{x} \leqslant \boldsymbol{y}$, если для всех компонент этих векторов вьполнено $x_{j} \leqslant y_{j}(j=1, \ldots, n)$, и $\boldsymbol{x}<\boldsymbol{y}$, если для всех компонент этих векторов вьполнено $x_{j}<y_{j}(j=1, \ldots, n)$. Аналогично определяются неравенства для вещественных матриц.

Лемма 1. Пусть $\alpha \boldsymbol{w} \leqslant \boldsymbol{A} \boldsymbol{w}$, дде $\alpha$ - неотрицательное число, $\boldsymbol{w}$ - ненулевой неотрицательный вектор и $\boldsymbol{A}$ - неотрицательная матрица. Тогда $\alpha \leqslant \operatorname{spr} \boldsymbol{A}$, причем если $\alpha \boldsymbol{w}<\boldsymbol{A w}$, то $\alpha<\operatorname{spr} \boldsymbol{A}$.

ДокАЗАТЕЛЬСТво первой части леммы дано в [3, с. 67, лемма 9.1], а справедливость второй части немедленно вытекает из неравенств $\alpha \boldsymbol{w}<\beta \boldsymbol{w} \leqslant \boldsymbol{A} w$, где $\beta>\alpha$ выбрано так, чтобы $\beta \boldsymbol{w} \leqslant \boldsymbol{A} w$ (при этом мы без ограничения обшности считаем вектор $\boldsymbol{w}$ положительным).

В статье систематически используется обозначение

$$
\|\boldsymbol{z}(\cdot)\| \|=\left(\int_{0}^{\omega}\|z(t)\|^{2} d t\right)^{1 / 2}
$$

для векторной функции $\boldsymbol{z}(t)$, суммируемой с квадратом на отрезке $[0, \omega]$. В книге [4, c. 19-22] дано геометрическое доказательство теоремы Йорка, опирающееся на свойства сферической индикатрисы фазовой кривой. Предлагаемое нами аналитическое доказательство основано на использовании хорошо известного неравенства Виртингера [5, с. 223], которое нам удобно записать в следующем виде

$$
\varkappa\|\boldsymbol{z}(\cdot)\| \leqslant\|\dot{z}(\cdot)\|, \quad \varkappa=\frac{2 \pi}{\omega} .
$$

Здесь $\boldsymbol{z}(t)$ - абсолютно непрерывная периодическая с периодом $\omega$ векторная функция, имеющая нулевое среднее значение и суммируемую с квадратом производную (на отрезке $[0, \omega])$. Доказьваемое с помошюю равенства Парсеваля неравенство (4) обрашается в равенство тогда и только тогда, когда $\boldsymbol{z}(t)$ есть линейная комбинация с векторными коэффишиентами функций $\cos \varkappa t$ и $\sin \varkappa t$ (или $\exp (i \varkappa t)$ и $\exp (-i \varkappa t))$. 
ТеоремА 1. Для периода $\omega$ периодического нестационарного решения $\boldsymbol{x}(t)$ системы (1), правые части которой удовлетворяют условию Липиича (3) с неотрицательной $(m \times m)$-матрицей $\boldsymbol{L}$, имеющей положстельный спектральный радиус, справедлива оченка снизу

$$
\omega \geqslant \frac{2 \pi}{\operatorname{spr} \boldsymbol{L}}
$$

ДоКАЗАТЕЛЬСТВО. По условию теоремы имеет место (2) и

$$
\dot{\boldsymbol{x}}_{j}(t)=\boldsymbol{g}_{j}\left(\boldsymbol{x}_{1}(t), \ldots, \boldsymbol{x}_{m}(t)\right), \quad t \in \mathbb{R}, \quad j=1, \ldots, m
$$

Рассмотрим разность

$$
\boldsymbol{z}(t)=\boldsymbol{x}(t+\triangle)-\boldsymbol{x}(t)
$$

где $\triangle$ - произвольное фиксированное число, несоизмеримое с периодом $\omega$. В силу автономности системы $(1)$ сдвиг $\boldsymbol{x}(t+\triangle)$ также является решением этой системы. Для нас важно, что ненулевая векторная функция $\boldsymbol{z}(t)$ имеет нулевое среднее значение.

Из (7) в силу (6) и условия Липшица (3) получаем неравенство

$$
\left\|\dot{z}_{j}(t)\right\| \leqslant \sum_{k=1}^{m} l_{j k}\left\|z_{k}(t)\right\|
$$

причем здесь и в написанных ниже неравенствах $j=1, \ldots, m$. По неравенству треугольника в $L_{2}$ имеем

$$
\left\|\sum_{k=1}^{m} l_{j k}\right\| z_{k}(\cdot)\|\| \leqslant \sum_{k=1}^{m} l_{j k}\left\|z_{k}(\cdot)\right\| \| .
$$

Поэтому из (8) и (9) находим, что

$$
\left\|\dot{z}_{j}(\cdot)\right\|\left|\leqslant \sum_{k=1}^{m} l_{j k}\right|\left\|z_{k}(\cdot) \mid\right\| .
$$

Так как по неравенству Виртингера (4)

$$
\varkappa\left\|z_{j}(\cdot)\left|\|\leqslant\| \dot{z}_{j}(\cdot)\right|\right\|
$$

то из (10) следует

$$
\varkappa\left\|z_{j}(\cdot)\left|\left\|\leqslant \sum_{k=1}^{m} l_{j k}\right\|\right| z_{k}(\cdot) \mid\right\| .
$$

Обозначим через $\boldsymbol{w}$ ненулевой неотрицательный вектор с компонентами $\left\|\mid \boldsymbol{z}_{j}(\cdot)\right\| \|, j=$ $1, \ldots, m$. По лемме 1 из неравенства $\varkappa \boldsymbol{w} \leqslant \boldsymbol{L} w$ вытекает оценка $\varkappa \leqslant \operatorname{spr} \boldsymbol{L}$, которая с точностью до обозначения совпадает с (5).

Коснемся вкратце вопроса об условиях обращения неравенства (5) в равенство. Периодическое нестационарное решение системы (1) назовем әкстремальным, если для 
него в $(5)$ имеет место знак равенства, т.е. $\varkappa=\operatorname{spr} \boldsymbol{L}$. Выясним сушествуют ли экстремальные решения и какими они обладают свойствами, ограничившись случаем, когда $\boldsymbol{L}$ - неразложимая матрица.

Если $\varkappa=\operatorname{spr} \boldsymbol{L}$, то в силу неразложимости матрицы $\boldsymbol{L}$ из оценки $\varkappa \boldsymbol{w} \leqslant \boldsymbol{L} w$ вытекает $\boldsymbol{\varkappa} \boldsymbol{w}=\boldsymbol{L} w$ и $\boldsymbol{w}>0$. Таким образом, $\varkappa$ является перроновым собственным значением матрицы $\boldsymbol{L}$, a $\boldsymbol{w}$ - соответствуюшим перроновым собственным вектором. Однако, для того чтобы в (12) имели место только знаки равенства, необходимо и достаточно, чтобы одновременно имели место только знаки равенства в $(8),(9)$ и (11).

Последнее в силу неравенства Виртингера (4) эквивалентно условию

$$
\boldsymbol{z}_{j}(t)=\boldsymbol{u}_{j} \cos \varkappa t+\boldsymbol{v}_{j} \sin \varkappa t, \quad j=1, \ldots, m
$$

где $\boldsymbol{u}_{j}$ и $\boldsymbol{v}_{j}$ - некоторые постоянные векторы. Равенство в (9) согласно строгой нормированности $L_{2}$ эквивалентно тому, что $\left\|z_{k}(t)\right\|$ между собой пропорциональны при $k=1, \ldots, m$ (при этом мы используем неразложимость матрицы $\boldsymbol{L}$ ). Таким образом, равенство в (8) эквивалентно условию $\left\|\dot{z}_{j}(t)\right\|=c_{j}\left\|z_{j}(t)\right\|$, где $c_{j}$ - некоторые положительные постоянные, $j=1, \ldots, m$. Согласно (13) последнее равенство эквивалентно тому, что ненулевые векторы $\boldsymbol{u}_{j}$ и $\boldsymbol{v}_{j}$ имеют одинаковую длину и ортогональны в $\boldsymbol{\theta} e-$ щественном смысле, т.е.

$$
\left\|\boldsymbol{u}_{j}\right\|=\left\|\boldsymbol{v}_{j}\right\|>0, \quad \operatorname{Re}\left(\boldsymbol{u}_{j}, \boldsymbol{v}_{j}\right)=0, \quad j=1, \ldots, m
$$

Из (13) и (14) после вычислений для экстремального решения получаем

$$
\boldsymbol{x}_{j}(t)=\boldsymbol{a}_{j} \cos \varkappa t+\boldsymbol{b}_{j} \sin \varkappa t+\boldsymbol{c}_{j}, \quad j=1, \ldots, m,
$$

где $\boldsymbol{a}_{j}, \boldsymbol{b}_{j}, \boldsymbol{c}_{j}$ - некоторые постоянные векторы, причем ненулевые $\boldsymbol{a}_{j}$ и $\boldsymbol{b}_{j}$ имеют одинаковую длину и ортогональны в вешественном смысле, т.е.

$$
\left\|\boldsymbol{a}_{j}\right\|=\left\|\boldsymbol{b}_{j}\right\|>0, \quad \operatorname{Re}\left(\boldsymbol{a}_{j}, \boldsymbol{b}_{j}\right)=0, \quad j=1, \ldots, m .
$$

Отсюда мы делаем важный вывод: если в вешественном случае хотя бы один из векторов $\boldsymbol{x}_{1}, \ldots, \boldsymbol{x}_{m}$ оказался одномерным (т.е. скаляром), то экстремальных решений не существует. Так будет, например, для системы

$$
\dot{x}_{j}=g_{j}\left(x_{1}, \ldots, x_{n}\right), \quad j=1, \ldots, n
$$

для которой условие Липшища принимает вид

$$
\left|g_{j}\left(x_{1}, \ldots, x_{n}\right)-g_{j}\left(y_{1}, \ldots, y_{n}\right)\right| \leqslant \sum_{k=1}^{n} l_{j k}\left|x_{k}-y_{k}\right|, \quad j=1, \ldots, n \text {. }
$$

Поэтому оценка (5) в этом частном случае не является точной и может быть улучшена. Нетрудно показать, что в комплексном случае или в вещественном, когда $d_{j} \geqslant 2$ при 
$j=1, \ldots, m$, всегда можно построить систему (1), удовлетворяющую условию Липшица (3) с матрицей $\boldsymbol{L}$, для которой (15) с (16) является экстремальным решением.

Подчеркнем связь между экстремальными периодическими колебаниями и спектральными характеристиками матрищы $\boldsymbol{L}$ : частота колебаний $\varkappa$ является перроновым собственньм значением матришы $\boldsymbol{L}$, а радиусы $r_{1}, \ldots, r_{m}$ окружностей, заданных параметрически в (15) с (16), равные общему значению $\left\|\boldsymbol{a}_{j}\right\|$ и $\left\|\boldsymbol{b}_{j}\right\|$, дают компонентыперронова собственного вектора $\boldsymbol{r}$ матрицы $\boldsymbol{L}: \varkappa \boldsymbol{r}=\boldsymbol{L} \boldsymbol{r}$.

Данное нами доказательство теоремы 1 без труда переносится на автономную систему нелинейных дифференциальных уравнений $p$-го порядка

$$
\boldsymbol{x}_{j}^{(p)}=\boldsymbol{g}_{j}\left(\boldsymbol{x}_{1}, \ldots, \boldsymbol{x}_{m}, \dot{\boldsymbol{x}}_{1}, \ldots, \dot{\boldsymbol{x}}_{m}, \ldots, \boldsymbol{x}_{1}^{(p-1)}, \ldots, \boldsymbol{x}_{m}^{(p-1)}\right), \quad j=1, \ldots, m,
$$

которую мы будем изучать или в $\mathbb{R}^{n} \times \mathbb{R}^{n} \times \cdots \times \mathbb{R}^{n}(p$ раз $)$ - вещественный случай, или в $\mathbb{C}^{n} \times \mathbb{C}^{n} \times \cdots \times \mathbb{C}^{n}(p$ раз) - комплексный случай. Для системы (17) сохраняются определения стационарного и нестационарного решений. Предполагается, что правые части системы (17) удовлетворяют условию Липшица в следующем виде:

$$
\begin{aligned}
& \| \boldsymbol{g}_{j}\left(\boldsymbol{x}_{1}, \ldots, \boldsymbol{x}_{m}, \dot{\boldsymbol{x}}_{1}, \ldots, \dot{\boldsymbol{x}}_{m}, \ldots, \boldsymbol{x}_{1}^{(p-1)}, \ldots, \boldsymbol{x}_{m}^{(p-1)}\right) \\
& \quad-\boldsymbol{g}_{j}\left(\boldsymbol{y}_{1}, \ldots, \boldsymbol{y}_{m}, \dot{\boldsymbol{y}}_{1}, \ldots, \dot{\boldsymbol{y}}_{m}, \ldots, \boldsymbol{y}_{1}^{(p-1)}, \ldots, \boldsymbol{y}_{m}^{(p-1)}\right) \| \\
& \leqslant \sum_{k=1}^{m} l_{j k}^{(0)}\left\|\boldsymbol{x}_{k}-\boldsymbol{y}_{k}\right\|+\sum_{k=1}^{m} l_{j k}^{(1)}\left\|\dot{\boldsymbol{x}}_{k}-\dot{\boldsymbol{y}}_{k}\right\|+\cdots+\sum_{k=1}^{m} l_{j k}^{(p-1)}\left\|\boldsymbol{x}_{k}^{(p-1)}-\boldsymbol{y}_{k}^{(p-1)}\right\|, \\
& j=1, \ldots, m,
\end{aligned}
$$

где $\boldsymbol{L}_{0}=\left(l_{j k}^{(0)}\right), \boldsymbol{L}_{1}=\left(l_{j k}^{(1)}\right), \ldots, \boldsymbol{L}_{p-1}=\left(l_{j k}^{(p-1)}\right)$ - некоторые неотрицательные $(m \times m)$ матрищы, сумма которых $\boldsymbol{L}_{0}+\boldsymbol{L}_{1}+\cdots+\boldsymbol{L}_{p-1}$ имеет положительньй спектральный радиус: $\operatorname{spr}\left(\boldsymbol{L}_{0}+\boldsymbol{L}_{1}+\cdots+\boldsymbol{L}_{p-1}\right)>0$. Последнее требование заведомо вьполнено, если упомянутая сумма есть положительная матрица или неотрицательная неразложимая матрица.

Если $m=1$, то система (17) преврашается в одно уравнение $p$-го порядка

$$
\boldsymbol{x}^{(p)}=\boldsymbol{g}\left(\boldsymbol{x}, \dot{\boldsymbol{x}}, \ldots, \boldsymbol{x}^{(p-1)}\right),
$$

для которого условие Липшица (18) записывается в виде

$$
\begin{aligned}
& \left\|\boldsymbol{g}\left(\boldsymbol{x}, \dot{\boldsymbol{x}}, \ldots, \boldsymbol{x}^{(p-1)}\right)-\boldsymbol{g}\left(\boldsymbol{y}, \dot{\boldsymbol{y}}, \ldots, \boldsymbol{y}^{(p-1)}\right)\right\| \\
& \quad \leqslant l_{0}\|\boldsymbol{x}-\boldsymbol{y}\|+l_{1}\|\dot{\boldsymbol{x}}-\dot{\boldsymbol{y}}\|+\cdots+l_{p-1}\left\|\boldsymbol{x}^{(p-1)}-\boldsymbol{y}^{(p-1)}\right\|,
\end{aligned}
$$

причем среди неотрицательных чисел $l_{0}, l_{1}, \ldots, l_{p-1}$ есть хотя бы одно положительное. Если $m=n$, то система (17) распадается на $n$ скалярных уравнений

$$
\boldsymbol{x}_{j}^{(p)}=\boldsymbol{g}_{j}\left(\boldsymbol{x}_{1}, \ldots, \boldsymbol{x}_{n}, \dot{\boldsymbol{x}}_{1}, \ldots, \dot{\boldsymbol{x}}_{n}, \ldots, \boldsymbol{x}_{1}^{(p-1)}, \ldots, \boldsymbol{x}_{n}^{(p-1)}\right), \quad j=1, \ldots, n
$$


для этой системы условие Липшица (18) кратко можно записать в форме

$$
\begin{aligned}
& \left|\boldsymbol{g}\left(\boldsymbol{x}, \dot{\boldsymbol{x}}, \ldots, \boldsymbol{x}^{(p-1)}\right)-\boldsymbol{g}\left(\boldsymbol{y}, \dot{\boldsymbol{y}}, \ldots, \boldsymbol{y}^{(p-1)}\right)\right| \\
& \quad \leqslant L_{0}|\boldsymbol{x}-\boldsymbol{y}|+L_{1}|\dot{\boldsymbol{x}}-\dot{\boldsymbol{y}}|+\cdots+L_{p-1}\left|\boldsymbol{x}^{(p-1)}-\boldsymbol{y}^{(p-1)}\right| .
\end{aligned}
$$

Под модулем $|\boldsymbol{x}|$ вектора $\boldsymbol{x}$ с компонентами $x_{1}, \ldots, x_{n}$ мы понимаем неотрицательньй вектор с компонентами $\left|x_{1}\right|, \ldots,\left|x_{n}\right|$. Аналогично определяется модуль $|\boldsymbol{A}|$ матрицы $\boldsymbol{A}$.

Возврашаясь к общему случаю, рассмотрим скалярное уравнение

$$
\varphi(u) \equiv \operatorname{spr}\left(\frac{\boldsymbol{L}_{0}}{u^{p}}+\frac{\boldsymbol{L}_{1}}{u^{p-1}}+\cdots+\frac{\boldsymbol{L}_{p-1}}{u}\right)=1, \quad 0<u<+\infty .
$$

ЛЕмма 2. Скалярная функиия $\varphi(u)$ положительна, непрерывна, убывает и, кроме того, $\varphi(u) \rightarrow+\infty$ при $0<u \rightarrow 0 u \varphi(u) \rightarrow 0$ при $u \rightarrow+\infty$.

ДокАЗАТЕЛЬСТВо. Положим

$$
\boldsymbol{L}(u)=\sum_{k=0}^{p-1} \frac{\boldsymbol{L}_{k}}{u^{p-k}}, \quad 0<u<+\infty .
$$

Так как при $1 \leqslant u<+\infty$ вьполнены оценки

$$
\frac{1}{u^{p}}\left(\sum_{k=0}^{p-1} \boldsymbol{L}_{k}\right) \leqslant \boldsymbol{L}(u) \leqslant \frac{1}{u}\left(\sum_{k=0}^{p-1} \boldsymbol{L}_{k}\right),
$$

то

$$
\frac{1}{u^{p}} \operatorname{spr}\left(\sum_{k=0}^{p-1} \boldsymbol{L}_{k}\right) \leqslant \varphi(u) \leqslant \frac{1}{u} \operatorname{spr}\left(\sum_{k=0}^{p-1} \boldsymbol{L}_{k}\right),
$$

отсюда вытекает и положительность $\varphi(u)$ и стремление к нулю при $u \rightarrow+\infty$. Аналогично исследуется поведение $\varphi(u)$ при $0<u \leqslant 1$. Покажем, что $\varphi$ убьвает, т.е.

$$
(0<) u<v \Longrightarrow \varphi(u)>\varphi(v) \text {. }
$$

Предварительно заметим, что если положить $\boldsymbol{L}(u)=\left(l_{j k}(u)\right)($ см. $(24))$, то либо $l_{j k}(u) \equiv 0$, либо $l_{j k}(u)>0$, причем $l_{j k}^{\prime}(u)<0$ при $0<u<+\infty$.

Пусть $\boldsymbol{L}(v) \boldsymbol{k}=\sigma \boldsymbol{k}$, где $\sigma \equiv \operatorname{spr} \boldsymbol{L}(v)=\varphi(v)>0$ и $0 \neq \boldsymbol{k} \geqslant 0$. Если $\boldsymbol{k}>0$, то в каждой строке матришы $\boldsymbol{L}(v)$ есть положительный элемент $l_{j k}(v)>0$. Так как $l_{j k}(u)>l_{j k}(v)$ для таких $j$ и $k$, то $\boldsymbol{L}(u) \boldsymbol{k}>\boldsymbol{L}(v) \boldsymbol{k}=\sigma \boldsymbol{k}$ и по лемме 1 получаем $\varphi(u)=\operatorname{spr} \boldsymbol{L}(u)>\sigma=\operatorname{spr} \boldsymbol{L}(v)=\varphi(v)$, т.е. имеет место (25).

В том случае, когда вектор $k$ имеет нулевые компоненты, выпишем номера $1 \leqslant i_{1}<$ $\cdots<i_{s} \leqslant n$ всех его положительных компонент $k_{i_{1}}>0, \ldots, k_{i_{s}}>0$. Рассмотрим подматрицу $\widehat{\boldsymbol{L}}$, стоящую на пересечении строк и столбцов с номерами $i_{1}, \ldots, i_{s}$ матрицы $\boldsymbol{L}$, и вектор $\hat{\boldsymbol{k}}$ с компонентами $k_{i_{1}}, \ldots, k_{i_{s}}$. Ясно, что $\widehat{\boldsymbol{L}}(v) \hat{\boldsymbol{k}}=\sigma \hat{\boldsymbol{k}}$ и $\hat{\boldsymbol{k}}>0$. По уже доказанному $\operatorname{spr} \widehat{\boldsymbol{L}}(u)>\operatorname{spr} \widehat{\boldsymbol{L}}(v)$, и так как $\operatorname{spr} \boldsymbol{L}(u) \geqslant \operatorname{spr} \widehat{\boldsymbol{L}}(u)$ и $\operatorname{spr} \widehat{\boldsymbol{L}}(v)=\operatorname{spr} \boldsymbol{L}(v)$, то $\varphi(u)=\operatorname{spr} \boldsymbol{L}(u)>\operatorname{spr} \boldsymbol{L}(v)=\varphi(v)$, чем и завершается доказательство свойства (25).

Из леммы 2 вытекает, что уравнение (23) имеет единственное положительное решение, которое мы обозначим $\varkappa_{0}$. Поэтому если для некоторого $\varkappa>0$ выполнено

$$
\varphi(\varkappa) \equiv \operatorname{spr}\left(\frac{\boldsymbol{L}_{0}}{\varkappa^{p}}+\frac{\boldsymbol{L}_{1}}{\varkappa^{p-1}}+\cdots+\frac{\boldsymbol{L}_{p-1}}{\varkappa}\right) \geqslant 1,
$$

то $\varkappa \leqslant \varkappa_{0}$. 
ТЕорема 2. Для периода $\omega$ периодического нестационарного решения $\boldsymbol{x}(t)$ системы (17), правые части которой удовлетворяют условию Липиица (18) с неотрицательными $(m \times m)$-матрицами $\boldsymbol{L}_{0}, \boldsymbol{L}_{1}, \ldots, \boldsymbol{L}_{p-1}$, спектральный радиус суммы которых положителен, справедлива оценка снизу

$$
\omega \geqslant \frac{2 \pi}{\varkappa_{0}}
$$

әде $\varkappa_{0}-$ единственный положительный корень уравнения (23).

ДокАЗАТЕЛЬСТВО. Перейдем от системы $m$ векторных уравнений $p$-го порядка (17) к системе $m p$ векторных уравнений первого порядка

$$
\begin{aligned}
& \dot{\boldsymbol{x}}_{1}=\boldsymbol{x}_{1}^{(1)}, \quad \dot{\boldsymbol{x}}_{2}=\boldsymbol{x}_{2}^{(1)}, \ldots, \quad \dot{\boldsymbol{x}}_{m}=\boldsymbol{x}_{m}^{(1)}, \\
& \boldsymbol{x}_{1}^{(p-2)}=\boldsymbol{x}_{1}^{(p-1)}, \boldsymbol{x}_{2}^{(p-2)}=\boldsymbol{x}_{2}^{(p-1)}, \ldots, \boldsymbol{x}_{m}^{(p-2)}=\boldsymbol{x}_{m}^{(p-1)}, \\
& \boldsymbol{x}_{1}^{(p-1)}=\boldsymbol{g}_{1}\left(\boldsymbol{x}_{1}, \ldots, \boldsymbol{x}_{m}, \boldsymbol{x}_{1}^{(1)}, \ldots, \boldsymbol{x}_{m}^{(1)}, \ldots, \boldsymbol{x}_{1}^{(p-1)}, \ldots, \boldsymbol{x}_{m}^{(p-1)}\right), \\
& \boldsymbol{x}_{m}^{(p-1)}=\boldsymbol{g}_{m}\left(\boldsymbol{x}_{1}, \ldots, \boldsymbol{x}_{m}, \boldsymbol{x}_{1}^{(1)}, \ldots, \boldsymbol{x}_{m}^{(1)}, \ldots, \boldsymbol{x}_{1}^{(p-1)}, \ldots, \boldsymbol{x}_{m}^{(p-1)}\right)
\end{aligned}
$$

Мы видим, что в силу (18) она удовлетворяет условию Липшища типа (3) с блочной матрицей

$$
\boldsymbol{L} \equiv\left(\begin{array}{ccccc}
0 & \boldsymbol{I} & 0 & \ldots & 0 \\
0 & 0 & \boldsymbol{I} & \ldots & 0 \\
\ldots & \ldots & \ldots & \ldots & \ldots \\
0 & 0 & 0 & \ldots & \boldsymbol{I} \\
\boldsymbol{L}_{0} & \boldsymbol{L}_{1} & \boldsymbol{L}_{2} & \ldots & \boldsymbol{L}_{p-1}
\end{array}\right)
$$

где $\boldsymbol{I}$ есть единичная $(m \times m)$-матрища.

Покажем, что для неотрицательной $(m p \times m p)$-матрицы $\boldsymbol{L}$ справедлива формула

$$
\operatorname{spr} \boldsymbol{L}=\varkappa_{0}
$$

где $\varkappa_{0}$, напомним, есть единственный положительньй корень уравнения (23). Итак, $\varkappa_{0}>0$ и

$$
\operatorname{spr}\left(\frac{\boldsymbol{L}_{0}}{\varkappa_{0}^{p}}+\frac{\boldsymbol{L}_{1}}{\varkappa_{0}^{p-1}}+\cdots+\frac{\boldsymbol{L}_{p-1}}{\varkappa_{0}}\right)=1 .
$$

Обозначим через $\boldsymbol{h}^{(0)}$ соответствующий неотрицательный собственньй вектор матрицы $\boldsymbol{L}\left(\varkappa_{0}\right)$ :

$$
\left(\frac{\boldsymbol{L}_{0}}{\varkappa_{0}^{p}}+\frac{\boldsymbol{L}_{1}}{\varkappa_{0}^{p-1}}+\cdots+\frac{\boldsymbol{L}_{p-1}}{\varkappa_{0}}\right) \boldsymbol{h}^{(0)}=\boldsymbol{h}^{(0)} .
$$

Тогда, полагая

$$
\boldsymbol{h}^{(1)}=\varkappa_{0} \boldsymbol{h}^{(0)}, \quad \boldsymbol{h}^{(2)}=\varkappa_{0}^{2} \boldsymbol{h}^{(0)}, \ldots, \quad \boldsymbol{h}^{(p-1)}=\varkappa_{0}^{p-1} \boldsymbol{h}^{(0)}
$$


в силу (31) получаем

$$
\begin{gathered}
\boldsymbol{h}^{(1)}=\varkappa_{0} \boldsymbol{h}^{(0)}, \quad \boldsymbol{h}^{(2)}=\varkappa_{0} \boldsymbol{h}^{(1)}, \ldots, \quad \boldsymbol{h}^{(p-1)}=\varkappa_{0} \boldsymbol{h}^{(p-2)} \\
\boldsymbol{L}_{0} \boldsymbol{h}^{(0)}+\boldsymbol{L}_{1} \boldsymbol{h}^{(1)}+\cdots+\boldsymbol{L}_{p-1} \boldsymbol{h}^{(p-1)}=\varkappa_{0} \boldsymbol{h}^{(p-1)}
\end{gathered}
$$

что коротко может быть записано в виде $\boldsymbol{L} \boldsymbol{h}=\varkappa_{0} \boldsymbol{h}$, где $\boldsymbol{h}=\operatorname{col}\left(\boldsymbol{h}^{(0)}, \boldsymbol{h}^{(1)}, \ldots, \boldsymbol{h}^{(p-1)}\right)$ (см. (29)). Так как $\boldsymbol{h}$ - ненулевой неотрицательный вектор, то из $\boldsymbol{L} \boldsymbol{h}=\varkappa_{0} \boldsymbol{h}$ в силу леммы 1 имеем spr $\boldsymbol{L} \geqslant \varkappa_{0}$.

Итак, спектральный радиус $\rho$ матрицы $\boldsymbol{L}$ положителен, и для доказательства формулы (30) нам осталось показать, что $\rho \leqslant \varkappa_{0}$. Пусть $\boldsymbol{h}$ - перронов собственный вектор матрицы $\boldsymbol{L}: \boldsymbol{L} \boldsymbol{h}=\rho \boldsymbol{h}$ и $0 \neq \boldsymbol{h} \geqslant 0$. Тогда, полагая $\boldsymbol{h}=\operatorname{col}\left(\boldsymbol{h}^{(0)}, \boldsymbol{h}^{(1)}, \ldots, \boldsymbol{h}^{(p-1)}\right)$, согласно (29) получаем

$$
\begin{gathered}
\boldsymbol{h}^{(1)}=\rho \boldsymbol{h}^{(0)}, \quad \boldsymbol{h}^{(2)}=\rho \boldsymbol{h}^{(1)}, \ldots, \quad \boldsymbol{h}^{(p-1)}=\rho \boldsymbol{h}^{(p-2)}, \\
\boldsymbol{L}_{0} \boldsymbol{h}^{(0)}+\boldsymbol{L}_{1} \boldsymbol{h}^{(1)}+\cdots+\boldsymbol{L}_{p-1} \boldsymbol{h}^{(p-1)}=\rho \boldsymbol{h}^{(p-1)}
\end{gathered}
$$

отсюда находим

$$
\begin{aligned}
\boldsymbol{h}^{(1)}= & \rho \boldsymbol{h}^{(0)}, \quad \boldsymbol{h}^{(2)}=\rho^{2} \boldsymbol{h}^{(0)}, \ldots, \quad \boldsymbol{h}^{(p-1)}=\rho^{p-1} \boldsymbol{h}^{(0)} \\
& \left(\boldsymbol{L}_{0}+\rho \boldsymbol{L}_{1}+\cdots+\rho^{p-1} \boldsymbol{L}_{p-1}\right) \boldsymbol{h}^{(0)}=\rho^{p} \boldsymbol{h}^{(0)}
\end{aligned}
$$

и потому

$$
\left(\frac{\boldsymbol{L}_{0}}{\rho^{p}}+\frac{\boldsymbol{L}_{1}}{\rho^{p-1}}+\cdots+\frac{\boldsymbol{L}_{p-1}}{\rho}\right) \boldsymbol{h}^{(0)}=\boldsymbol{h}^{(0)} .
$$

Вектор $\boldsymbol{h}^{(0)}$ - ненулевой неотрицательный вектор, причем $\boldsymbol{L}(\rho) \boldsymbol{h}^{(0)}=\boldsymbol{h}^{(0)}$ согласно (32). По лемме 1 получаем $\varphi(\rho) \equiv \operatorname{spr} \boldsymbol{L}(\rho) \geqslant 1$, т.е. имеет место неравенство (26) и, значит, $(0<) \rho \leqslant \varkappa_{0}$. Формула $(30)$ полностью доказана.

Наш успех, возможно, объясняется тем, что

$$
\operatorname{det}(\lambda \boldsymbol{J}-\boldsymbol{L}) \equiv \operatorname{det}\left(\lambda^{p} \boldsymbol{I}-\left(\boldsymbol{L}_{0}+\lambda \boldsymbol{L}_{1}+\cdots+\lambda^{p-1} \boldsymbol{L}_{p-1}\right)\right)
$$

где $\boldsymbol{J}$ - единичная $(m p \times m p)$-матрица $(\mathrm{a} \boldsymbol{I}$ - единичная $(m \times m)$-матрица). Мы видим, что матрища $\boldsymbol{L}$ представляет собой блочньй вариант сопровождающей матрицы Фробениуса для матричного многочлена $\lambda^{p} \boldsymbol{I}-\left(\boldsymbol{L}_{0}+\lambda \boldsymbol{L}_{1}+\cdots+\lambda^{p-1} \boldsymbol{L}_{p-1}\right)$.

Пусть $\boldsymbol{x}_{1}(t), \ldots, \boldsymbol{x}_{m}(t)$ - периодическое с периодом $\omega$ нестационарное решение системы (17). Тогда $\boldsymbol{x}_{1}(t), \ldots, \boldsymbol{x}_{m}(t), \dot{\boldsymbol{x}}_{1}(t), \ldots, \dot{\boldsymbol{x}}_{m}(t), \ldots, \boldsymbol{x}_{1}^{(p-1)}(t), \ldots, \boldsymbol{x}_{m}^{(p-1)}(t)$ есть периодическое с периодом $\omega$ нестационарное решение системы (28). По теореме 1 имеет место оценка $\omega \geqslant 2 \pi / \operatorname{spr} \boldsymbol{L}$, где $\boldsymbol{L}$ - это матрица (29). Согласно формуле (30) из последнего неравенства приходим к оценке (27).

Вопрос о точности оценки (27) и свойствах экстремальных решений можно изучить так же, как это сделано при $p=1$. Экстремальное решение по-прежнему имеет вид (15), 
но свойства (16) могут отсутствовать (если, например, в системе (17) встречаются производные только четного или только нечетного порядка).

Отметим только, что векторная функция $\boldsymbol{x}(t)=\exp \left(i \varkappa_{0} t\right) \boldsymbol{h}^{(0)}$, где $\boldsymbol{L}\left(\varkappa_{0}\right) \boldsymbol{h}^{(0)}=\boldsymbol{h}^{(0)}$, является экстремальным решением системы $(21)$, коротко записанной в виде

$$
\boldsymbol{x}^{(p)}=\boldsymbol{A}_{0} \boldsymbol{x}+\boldsymbol{A}_{1} \dot{\boldsymbol{x}}+\cdots+\boldsymbol{A}_{p-1} \boldsymbol{x}^{(p-1)},
$$

где

$$
\boldsymbol{A}_{0}=i^{p} \boldsymbol{L}_{0}, \quad \boldsymbol{A}_{1}=i^{p-1} \boldsymbol{L}_{1}, \quad \ldots, \quad \boldsymbol{A}_{p-1}=i \boldsymbol{L}_{p-1} .
$$

Taк как

$$
\left|\boldsymbol{A}_{0}\right|=\boldsymbol{L}_{0}, \quad\left|\boldsymbol{A}_{1}\right|=\boldsymbol{L}_{1}, \ldots, \quad\left|\boldsymbol{A}_{p-1}\right|=\boldsymbol{L}_{p-1},
$$

то условие Липшица (22) выполнено очевидным образом.

\section{СПИСОК ЦИТИРОВАННОЙ ЛИТЕРАТУРЫ}

[1] Гантмахер Ф.Р. Теория матриц. М.: Наука, 1967.

[2] Jorke J. A. Periods of periodic solutions and the Lipschitz constants // Proc. Amer. Math. Soc. 1964. V. 22. P. 509-512.

[3] Красносельский М. А., Лифшиц Е. А., Соболев А. В. Позитивные линейные системы. М.: Наука, 1985.

[4] Крюков Б. И. Вынужденные колебания существенно нелинейных систем. М.: Машиностроение, 1984.

[5] Харди Г.Г., Литтльвуд Д. Е., Полиа Г. Неравенства. М.: ИЛ, 1948.

Воронежский государственный университет 